\title{
ON THE MAILLET-BAKER CONTINUED FRACTIONS
}

\author{
BORIS ADAMCZEWSKI, YANN BUGEAUD
}

\begin{abstract}
We use the Schmidt Subspace Theorem to establish the transcendence of a class of quasi-periodic continued fractions. This improves earlier works of Maillet and of A. Baker. We also improve an old result of Davenport and Roth on the rate of increase of the denominators of the convergents to any real algebraic number.
\end{abstract}

\section{INTRODUCTION}

A central question in Diophantine approximation is concerned with how algebraic numbers can be approximated by rationals. This problem is intimately connected with the behaviour of their continued fraction expansion. In particular, it is widely believed that the continued fraction expansion of any irrational algebraic number $\xi$ either is eventually periodic (and we know that this is the case if, and only if, $\xi$ is a quadratic irrational), or it contains arbitrarily large partial quotients. Apparently, this problem was first considered by Khintchine in [1]. (we also refer the reader to 3, 21, 22 for surveys including a discussion on this subject). Some speculations about the randomness of the continued fraction expansion of algebraic numbers of degree at least three have later been made by Lang [12. However, one shall admit that our knowledge on this topic is up to now very limited.

A first step consists in providing explicit examples of transcendental continued fractions. The first result of this type goes back to the pioneering work of Liouville [14, who constructed transcendental real numbers with a very fast growing sequence of partial quotients. Subsequently, various authors used deeper transcendence criteria from Diophantine approximation to construct other classes of transcendental continued fractions. Of particular interest is the work of Maillet [15] (see also Section 34 of Perron [17]), who was the first to give explicit examples of transcendental continued fractions with bounded partial quotients. His work has later been carried on by A. Baker [4, 5].

More precisely, Maillet proved that if $\mathbf{a}=\left(a_{n}\right)_{n \geq 0}$ is a non-eventually periodic sequence of positive integers, and if there are infinitely many positive integers $n$ such that

$$
a_{n}=a_{n+1}=\ldots=a_{n+\lambda(n)-1},
$$

then the real number $\xi=\left[a_{0} ; a_{1}, a_{2}, \cdots\right]$ is transcendental, as soon as $\lambda(n)$ is larger than a certain function of the denominator of the $n$-th convergent to $\xi$. Actually, the result of Maillet is more general and also includes the case of repetitions of blocks of consecutive partial quotients (see Section 2). His proof is based on a general form of the Liouville inequality which 
limits the approximation of algebraic numbers by quadratic irrationals. Indeed, under the previous assumption, the quadratic irrational real numbers $\xi_{n}$, defined as having the eventually periodic continued fraction expansion $\left[a_{0} ; a_{1}, \cdots, a_{n-1}, a_{n}, a_{n}, a_{n}, \ldots\right]$, provide infinitely many 'too good' approximations to $\xi$.

Not surprisingly, the breakthrough made by Roth in his 1955 seminal paper 18 leads to an improvement of this result. Indeed, Baker 4 used in 1962 the Roth theorem for number fields obtained by LeVeque [13] to strongly improve upon the results of Maillet and make them more explicit. His main idea was to remark that when infinitely many of the quadratic approximations found by Maillet lie in a same quadratic number field, one can favourably replace the use of the Liouville inequality by the one of LeVeque's Theorem.

The purpose of the present paper is to improve the results obtained by Baker in 4, that are recalled in Section 2. Our approach rests on the Schmidt Subspace Theorem, but we follow a rather different way than the one previously considered by Maillet and by Baker. Our results are stated in Section 3 and proved in Section 6. Section 4 is devoted to the improvement of an old result of Davenport and Roth 9 on the rate of increase of the denominators of the convergents to any real algebraic number. It is the key point for the proof of Theorem 3.1 below, and is also of independent interest. Auxiliary results are gathered in Section 5 .

\section{EARLIER RESULTS}

Throughout the present paper, we keep the following notation. Let $\mathbf{a}=$ $\left(a_{n}\right)_{n \geq 0}$ be a sequence of positive integers, that is not eventually periodic. Let $\left(n_{k}\right)_{k \geq 0}$ be an increasing sequence of positive integers. Let $\left(\lambda_{k}\right)_{k \geq 0}$ and $\left(r_{k}\right)_{k \geq 0}$ be sequences of positive integers. Assume that for any non-negative integer $k$, we have $n_{k+1} \geq n_{k}+\lambda_{k} r_{k}$ and

$$
a_{m+r_{k}}=a_{m} \text { for } n_{k} \leq m \leq n_{k}+\left(\lambda_{k}-1\right) r_{k}-1,
$$

and consider the real number $\xi$ defined by

$$
\xi=\left[a_{0} ; a_{1}, a_{2}, \ldots, a_{n}, \ldots\right] .
$$

Then, $\xi$ has a quasi-periodic continued fraction expansion in the following sense: for any positive integer $k$, a block of $r_{k}$ consecutive partial quotients is repeated $\lambda_{k}$ times, such a repetition occurring just after the $\left(n_{k}-1\right)$-th partial quotient.

In [4, Baker established three theorems, which strongly improved the pioneering work of Maillet. The first one is very general.

Theorem 2.1 (A. Baker). With the previous notation, let us assume that

$$
\limsup _{k \rightarrow \infty} \frac{r_{k}}{n_{k}}<+\infty
$$

and

$$
\limsup _{k \rightarrow \infty} \frac{\left(\log \lambda_{k}\right)\left(\log n_{k}\right)^{1 / 2}}{n_{k}}=+\infty .
$$

Then, the real number $\xi$ is transcendental. 
Actually, it is not difficult to modify Baker's proof of Theorem 2.1]in order to get rid of the assumption (2). Notice also a related result due to Mignotte [16]. Under the assumption that the sequence $\mathbf{a}$ is bounded, condition (3) can be considerably relaxed.

Theorem 2.2 (A. Baker). Let $A \geq 2$ be an integer. Let $\mathbf{a}$ be a sequence of integers at most equal to $A$ that satisfy (11) for a bounded sequence $\left(r_{k}\right)_{k \geq 0}$. Assume that

$$
\limsup _{k \rightarrow \infty} \frac{\lambda_{k}}{n_{k}}>B=B(A)
$$

where $B$ is defined by

$$
B=2\left(\frac{\log \left(\left(A+\sqrt{A^{2}+4}\right) / 2\right)}{\log ((1+\sqrt{5}) / 2)}\right)-1 .
$$

Then, the real number $\xi$ is transcendental.

First, we remark that $B(A)$ increases with $A$ and that $\lim _{A \rightarrow \infty} B(A)=$ $+\infty$. The smallest value, obtained for $A=2$, is $B(2) \simeq 2.66 \ldots$ Let us also note that, when one only knows that the sequence $\mathbf{a}$ is bounded, but without having any explicit bound, the stronger assumption

$$
\limsup _{k \rightarrow \infty} \frac{\lambda_{k}}{n_{k}}=+\infty
$$

is required to apply Theorem 2.2 ,

One of the difficulties in the proof of Theorem 2.2 is that one needs a precise estimate for the growth of the sequence of the denominators of the convergents to $\xi$. This in particular explains why, in this result, the value of $B$ depends on $A$. However, for a more restricted class of quasiperiodic continued fractions, that we present now, Baker 4] partly succeeded in overcoming this difficulty.

Theorem 2.3 (A. Baker). Let us consider the quasi-periodic continued fraction

$$
\xi=[a_{0} ; a_{1}, \ldots, a_{n_{0}-1}, \underbrace{a_{n_{0}}, \ldots, a_{n_{0}+r_{0}-1}}_{\lambda_{0}}, \underbrace{a_{n_{1}}, \ldots, a_{n_{1}+r_{1}-1}}_{\lambda_{1}}, \ldots],
$$

where the notation implies that $n_{k+1}=n_{k}+\lambda_{k} r_{k}$ and the $\lambda$ 's indicate the number of times a block of partial quotients is repeated. Let us assume that the sequences $\left(a_{n}\right)_{n \geq 0}$ and $\left(r_{k}\right)_{k \geq 0}$ are both bounded, that $\left(a_{n}\right)_{n \geq 0}$ is not ultimately periodic, and that

$$
\liminf _{k \rightarrow \infty} \frac{\lambda_{k+1}}{\lambda_{k}}>2 .
$$

Then, the real number $\xi$ is transcendental.

As a typical example of such continued fractions, Baker considered at the end of [4] the following family of real numbers:

$$
\xi_{a, b}=[0 ; \underbrace{a, a, \ldots, a}_{\lambda_{0}}, \underbrace{b, b, \ldots, b}_{\lambda_{1}}, \underbrace{a, a, \ldots, a}_{\lambda_{2}}, b, b, \ldots],
$$


where $a$ and $b$ denote distinct positive integers. In the very particular case where $a=1$ and $b=2$, Baker improved Theorem 2.3 by showing that $\xi$ is a transcendental number as soon as $\liminf _{k \rightarrow \infty}\left(\lambda_{k+1} / \lambda_{k}\right)>1.72$. Unfortunately, Baker's approach does not enable us to replace 2 by a constant smaller than $\sqrt{2} \simeq 1,41$ in Inequality (5), even for the specific examples considered in ([6).

\section{MAin RESUlts}

We present here our main results which improve the three theorems due to Baker mentioned in the previous Section.

The first of Baker's results, namely Theorem 2.1. heavily rests on an upper bound due to Davenport and Roth [9] (see (13) below) for the rate of increase of the denominators of the convergents to any real algebraic number. Our improvement of (13) stated in Theorem 4.1 below allows us to get the following strengthening of Theorem 2.1

Theorem 3.1. Let $\mathbf{a}=\left(a_{n}\right)_{n \geq 0}$ be a sequence of positive integers, which satisfies (1) and is not ultimately periodic. Assume that

$$
\limsup _{k \rightarrow \infty} \frac{\log \lambda_{k}}{n_{k}^{\varepsilon+2 / 3}}=+\infty
$$

holds for some $\varepsilon>0$. Then, the real number $\xi=\left[a_{0} ; a_{1}, a_{2}, \ldots, a_{n}, \ldots\right]$ is transcendental.

In order to improve the two other results quoted in Section 2 it is tempting to try to apply the powerful Schmidt Subspace Theorem (see Section 5) instead of the result of LeVeque mentioned in the Introduction. For instance, the authors of [2] recently improved Theorem 2.3 via the Subspace Theorem, but only in the particular case given in (6), for which they reached the bound $\sqrt{2}$ (instead of 2), independently of the values of the distinct positive integers $a$ and $b$. See also related results by Davison [8].

Quite surprisingly, a different application of the Subspace Theorem based on the mirror formula (see Lemma $\mathbf{5 . 4}$ for a definition) allows us to considerably relax the assumptions of two of the transcendence criteria obtained by Baker. Our main result can be stated as follows.

Theorem 3.2. Let $\mathbf{a}=\left(a_{n}\right)_{n \geq 0}$ be a sequence of positive integers, which satisfies (11) and is not ultimately periodic. Let $\left(p_{n} / q_{n}\right)_{n \geq 1}$ denote the sequence of convergents to the real number

$$
\xi=\left[a_{0} ; a_{1}, a_{2}, \ldots, a_{n}, \ldots\right] .
$$

Assume that the sequence $\left(q_{n}^{1 / n}\right)_{n \geq 1}$ is bounded (which is in particular the case when the sequence $\mathbf{a}$ is bounded), and that

$$
\limsup _{k \rightarrow \infty} \frac{\lambda_{k}}{n_{k}}>0
$$

Then, the real number $\xi$ is transcendental. 
Unlike in Theorem 2.2, the transcendence condition obtained in Theorem 3.2 does not require neither that the partial quotients of the real number $\xi$ are bounded, nor that the lengths of the blocks which are repeated are bounded. Furthermore, we point out that the assumption 'the sequence $\left(q_{n}^{1 / n}\right)_{n \geq 1}$ is bounded' is satisfied by almost all real numbers. If one follows Baker's proof of Theorem 2.1 under this additional assumption, it is easily seen that one gets a much weaker version of Theorem 3.2. namely with the condition (8) being replaced by (4).

The proof of Theorem 3.2 splits into two parts. In the first part, we develop a new application of the Schmidt Subspace Theorem, based on Lemma 5.4 below. This is the main novelty of the present paper and it allows us to deal e.g. with real numbers $\xi$ satisfying the assumption of Baker's Theorem 2.2. The second part is far much easier.

As a direct corollary of Theorem 3.2 we obtain the following improvement of Theorem 2.3.

Corollary 3.3. Let us consider the quasi-periodic continued fraction

$$
\xi=[a_{0} ; a_{1}, \ldots, a_{n_{0}-1}, \underbrace{a_{n_{0}}, \ldots, a_{n_{0}+r_{0}-1}}_{\lambda_{0}}, \underbrace{a_{n_{1}}, \ldots, a_{n_{1}+r_{1}-1}}_{\lambda_{1}}, \ldots],
$$

where the notation implies that $n_{k+1}=n_{k}+\lambda_{k} r_{k}$ and the $\lambda$ 's indicate the number of times a block of partial quotients is repeated. Denote by $\left(p_{n} / q_{n}\right)_{n \geq 0}$ the sequence of the convergents to $\xi$. Assume that the sequences $\left(q_{n}^{1 / n}\right)_{n \geq 0}$ and $\left(r_{k}\right)_{k \geq 0}$ are bounded, that $\left(a_{n}\right)_{n \geq 0}$ is not ultimately periodic, and that

$$
\liminf _{k \rightarrow \infty} \frac{\lambda_{k+1}}{\lambda_{k}}>1
$$

Then, the real number $\xi$ is transcendental.

Finally, we mention that applying the Schmidt Subspace Theorem in a similar way as in our previous work [1] allows us to get rid of the assumptions on the sequences $\left(a_{n}\right)_{n \geq 0}$ and $\left(r_{k}\right)_{k \geq 0}$ in Theorem 2.3.

Theorem 3.4. Let us consider the quasi-periodic continued fraction

$$
\xi=[a_{0} ; a_{1}, \ldots, a_{n_{0}-1}, \underbrace{a_{n_{0}}, \ldots, a_{n_{0}+r_{0}-1}}_{\lambda_{0}}, \underbrace{a_{n_{1}}, \ldots, a_{n_{1}+r_{1}-1}}_{\lambda_{1}}, \ldots] .
$$

Assume that the sequence $\left(a_{n}\right)_{n \geq 0}$ is not ultimately periodic, and that

$$
\liminf _{k \rightarrow \infty} \frac{\lambda_{k+1}}{\lambda_{k}}>2 \text {. }
$$

Then, the real number $\xi$ is transcendental.

\section{An improvement of a Result of Davenport and Roth}

Throughout the present Section (which can be read independently of the rest of the paper), $\xi$ denotes an arbitrary irrational, real algebraic number and $\left(p_{n} / q_{n}\right)_{n \geq 1}$ always denotes the sequence of its convergents. The rate of growth of $\left(q_{n}\right)_{n \geq 1}$ is at least exponential, as immediately follows from the theory of continued fraction, see Lemma 5.3 below. Our purpose is to 
estimate it from above. It is well known that, if $\xi$ is quadratic, then there exists a real number $C(\xi)$, depending only on $\xi$, such that $q_{n}^{1 / n} \leq C(\xi)$ for any $n \geq 1$. It is widely believed that $\left(q_{n}^{1 / n}\right)_{n \geq 1}$ also remains bounded if the degree of $\xi$ is greater than two. However, we seem to be very far away from a proof (or a disproof).

The first general upper estimate for the rate of increase of $\left(q_{n}\right)_{n>1}$ follows from the Liouville inequality, saying that any algebraic number of degree $d$ cannot be approximated by rationals at an order greater than $d$. Using this result, we easily get that

$$
\log \log q_{n} \ll n .
$$

Throughout the present Section, all the constants implied by $\ll$ depend only on $\xi$.

Let $\delta$ be a positive real number. In 1955, Roth [18] proved that the set of solutions to the inequality

$$
\left|\xi-\frac{p}{q}\right|<\frac{1}{q^{2+\delta}}
$$

in integers $p, q$ with $\operatorname{gcd}(p, q)=1$ and $q>0$, is finite. In his joint work with Davenport 9], some steps from [18] were made more explicit in order to get an upper estimate for the cardinality $\mathcal{N}(\xi, \delta)$ of this set. In particular, Davenport and Roth 9 established that, for $\delta \leq 1 / 3$, there exist positive constants $c_{1}$ and $c_{2}$, depending only on $\xi$, such that

$$
\mathcal{N}(\xi, \delta) \leq c_{1} \exp \left\{c_{2} \delta^{-2}\right\} .
$$

They further derived from (12) an improvement of (11), namely the upper estimate

$$
\log \log q_{n} \ll \frac{n}{\sqrt{\log n}} .
$$

Bombieri and van der Poorten [6] were the first who established an upper bound for $\mathcal{N}(\xi, \delta)$ which is polynomial in $\delta^{-1}$. A slight sharpening has subsequently been obtained by Evertse, who proved at the end of Section 6 of [10] that, for $\delta<1$, there exists a positive constant $c_{3}$, depending only on $\xi$, such that

$$
\mathcal{N}(\xi, \delta) \leq c_{3} \delta^{-3}\left(1+\log \delta^{-1}\right)^{2} .
$$

Any qualitative improvement of (12) yields an improvement of (13). In particular, if we insert (14) instead of (12) in Davenport and Roth's proof of (13), we get the upper estimate

$$
\log \log q_{n} \ll n^{3 / 4} \sqrt{\log n} .
$$

It turns out that a suitable modification of the argument used by Davenport and Roth allows us to derive from (14) a much better result than (15).

Theorem 4.1. Let $\xi$ be an arbitrary irrational, real algebraic number and let $\left(p_{n} / q_{n}\right)_{n \geq 1}$ denote the sequence of its convergents. Then, for any $\varepsilon>0$, there exists a constant $c_{4}$, depending only on $\xi$ and $\varepsilon$, such that

$$
\log \log q_{n} \leq c_{4} n^{2 / 3+\varepsilon} .
$$


As an immediate corollary, we get a transcendence criterion for real numbers whose convergents have very large denominators.

Corollary 4.2. Let $\theta$ be an irrational, real number and let $\left(r_{n} / s_{n}\right)_{n \geq 1}$ denote the sequence of its convergents. If there exists a positive real number $\varepsilon$ such that

$$
\limsup _{n \rightarrow+\infty} \frac{\log \log s_{n}}{n^{2 / 3+\varepsilon}}=+\infty,
$$

then $\theta$ is transcendental.

Corollary 4.2 is the key point for the proof of Theorem 3.1

Proof of Theorem 4.1. The basic idea is to introduce more parameters in the proof of Theorem 3 of 9 . Recall that we have

$$
\left|\xi-\frac{p_{n}}{q_{n}}\right|<\frac{1}{q_{n} q_{n+1}},
$$

for any $n \geq 1$. Let $k \geq 1$ be an integer and $\delta_{1}, \ldots, \delta_{k}$ be real numbers with $0<\delta_{1}<\delta_{2}<\ldots<\delta_{k}<1$, that will be selected later on.

It is convenient to introduce a positive real number $\nu>1$ such that $\mathcal{N}(\xi, \delta) \ll \delta^{-\nu}$ holds for any $\delta$ with $0<\delta<1$. In view of (14), we can take for $\nu$ any real number strictly larger than 3 .

Let $N$ be a (sufficiently large) integer and put $\mathcal{S}_{0}=\{1,2, \ldots, N\}$. For $j=1, \ldots, k$, let $\mathcal{S}_{j}$ denote the set of positive integers $n$ in $\mathcal{S}_{0}$ such that $q_{n+1}>q_{n}^{1+\delta_{j}}$. Observe that $\mathcal{S}_{0} \supset \mathcal{S}_{1} \supset \ldots \supset \mathcal{S}_{k}$. It follows from (16) that, for any $n$ in $\mathcal{S}_{j}$, the convergent $p_{n} / q_{n}$ gives a solution to

$$
\left|\xi-\frac{p}{q}\right|<\frac{1}{q^{2+\delta_{j}}} .
$$

Consequently, the cardinality of $\mathcal{S}_{j}$ is at most $\mathcal{N}\left(\xi, \delta_{j}\right)$, thus it is $\ll \delta_{j}^{-\nu}$.

Write

$$
\mathcal{S}_{0}=\left(\mathcal{S}_{0} \backslash \mathcal{S}_{1}\right) \cup\left(\mathcal{S}_{1} \backslash \mathcal{S}_{2}\right) \cup \ldots \cup\left(\mathcal{S}_{k-1} \backslash \mathcal{S}_{k}\right) \cup \mathcal{S}_{k} .
$$

Let $j$ be an integer with $1 \leq j \leq k$. The cardinality of $\mathcal{S}_{0} \backslash \mathcal{S}_{1}$ is obviously bounded by $N$ and, if $j \geq 2$, the cardinality of $\mathcal{S}_{j-1} \backslash \mathcal{S}_{j}$ is $\ll \delta_{j-1}^{-\nu}$. Furthermore, for any $n$ in $\mathcal{S}_{j-1} \backslash \mathcal{S}_{j}$, we get

$$
\frac{\log q_{n+1}}{\log q_{n}} \leq 1+\delta_{j}
$$

Denoting by $d$ the degree of $\xi$, we infer from (16) and the Liouville inequality that

$$
\frac{\log q_{n+1}}{\log q_{n}} \leq d
$$

holds for every sufficiently large integer $n$ in $\mathcal{S}_{k}$. Combining these estimates with the fact that $\mathcal{S}_{k}$ has $\ll \delta_{k}^{-\nu}$ elements, we obtain that

$$
\log q_{N} \ll \frac{\log q_{N}}{\log q_{N-1}} \times \frac{\log q_{N-1}}{\log q_{N-2}} \times \ldots \times \frac{\log q_{3}}{\log q_{2}} \ll\left(1+\delta_{1}\right)^{N} \prod_{j=2}^{k}\left(1+\delta_{j}\right)^{\delta_{j-1}^{-\nu}} d^{\delta_{k}^{-\nu}} .
$$


Taking the logarithm, we get

$$
\log \log q_{N} \ll N \log \left(1+\delta_{1}\right)+\sum_{j=2}^{k} \delta_{j-1}^{-\nu} \log \left(1+\delta_{j}\right)+\delta_{k}^{-\nu} .
$$

We now select $\delta_{1}, \ldots, \delta_{k}$. For $j=1, \ldots, k$, set

$$
\delta_{j}=N^{-\left(\nu^{k}-\nu^{j-1}\right) /\left(\nu^{k+1}-1\right)} .
$$

We check that $0<\delta_{1}<\ldots<\delta_{k}<1$, and we easily infer from (17) that

$$
\log \log q_{N} \ll k N^{\left(\nu^{k+1}-\nu^{k}\right) /\left(\nu^{k+1}-1\right)}=k N^{(\nu-1) /\left(\nu-\nu^{-k}\right)} .
$$

By (11), we may assume that $\varepsilon \leq 1 / 3$. In view of (14), we can take $\nu=$ $3 /(1-\varepsilon)$. Choosing then for $k$ the smallest integer greater than $\log \varepsilon^{-1}$, we get from (18) that

$$
\log \log q_{N} \ll\left(\log \varepsilon^{-1}\right) N^{\varepsilon+2 / 3},
$$

as claimed.

\section{AuXiliary Results}

Our Theorems 3.2 and 3.4 rest on the Schmidt Subspace Theorem [19] (see also [20]), that we recall now.

Theorem 5.1 (W. M. Schmidt). Let $m \geq 2$ be an integer. Let $L_{1}, \ldots, L_{m}$ be linearly independent linear forms in $\mathbf{x}=\left(x_{1}, \ldots, x_{m}\right)$ with algebraic coefficients. Let $\varepsilon$ be a positive real number. Then, the set of solutions $\mathbf{x}=\left(x_{1}, \ldots, x_{m}\right)$ in $\mathbb{Z}^{m}$ to the inequality

$$
\left|L_{1}(\mathbf{x}) \ldots L_{m}(\mathbf{x})\right| \leq\left(\max \left\{\left|x_{1}\right|, \ldots,\left|x_{m}\right|\right\}\right)^{-\varepsilon}
$$

lies in finitely many proper subspaces of $\mathbb{Q}^{m}$.

For the reader convenience, we recall here some classical results from the theory of continued fractions, whose proofs can be found for example in the book of Perron [17].

Lemma 5.2. Let $\xi=\left[a_{0} ; a_{1}, a_{2}, \cdots\right]$ and $\eta=\left[b_{0} ; b_{1}, b_{2}, \cdots\right]$ be real numbers. Let $n \geq 1$ such that $a_{j}=b_{j}$ for any $j=0, \ldots, n$. We then have $|\xi-\eta| \leq q_{n}^{-2}$, where $q_{n}$ denotes the denominator of the $n$-th convergent to $\xi$.

Lemma 5.3. Let $\left(a_{n}\right)_{n \geq 0}$ be a sequence of positive integers at most equal to $M$, let $n$ be a positive integer and set $p_{n} / q_{n}=\left[a_{0} ; a_{1}, a_{2}, \ldots, a_{n}\right]$. Then, we have

$$
\sqrt{2}^{(n-1)} \leq q_{n} \leq(M+1)^{n} .
$$

The following innocent-looking formula appears to be the key point in the proof of Theorem 3.2. In what follows, Equality (19) will be referred to as the mirror formula.

Lemma 5.4. Let $\xi=\left[a_{0} ; a_{1}, a_{2}, \cdots\right]$ be a real number with convergents $\left(p_{n} / q_{n}\right)_{n \geq 1}$. Then, for any $n \geq 2$, we have

$$
\frac{q_{n}}{q_{n-1}}=\left[a_{n} ; a_{n-1}, \ldots, a_{1}\right] .
$$


For positive integers $a_{1}, \ldots, a_{m}$, denote by $K_{m}\left(a_{1}, \ldots, a_{m}\right)$ the denominator of the rational number $\left[0 ; a_{1}, \ldots, a_{m}\right]$. It is commonly called a continuant.

Lemma 5.5. For any positive integers $a_{1}, \ldots, a_{m}$ and any integer $k$ with $1 \leq k \leq m-1$, we have

$$
\begin{gathered}
K_{m}\left(a_{1}, \ldots, a_{m}\right)=K_{m}\left(a_{m}, \ldots, a_{1}\right) \\
K_{k}\left(a_{1}, \ldots, a_{k}\right) \cdot K_{m-k}\left(a_{k+1}, \ldots, a_{m}\right) \leq K_{m}\left(a_{1}, \ldots, a_{m}\right) \\
\leq 2 K_{k}\left(a_{1}, \ldots, a_{k}\right) \cdot K_{m-k}\left(a_{k+1}, \ldots, a_{m}\right)
\end{gathered}
$$

and

$$
\begin{aligned}
\frac{1}{2} K_{m}\left(a_{k}, \ldots, a_{m}, a_{1}, \ldots, a_{k-1}\right) & \leq K_{m}\left(a_{1}, \ldots, a_{m}\right) \\
& \leq 2 K_{m}\left(a_{k}, \ldots, a_{m}, a_{1}, \ldots, a_{k-1}\right) .
\end{aligned}
$$

We finish our series of lemmas by an immediate consequence of Roth's theorem.

Lemma 5.6. Let $\left(p_{n} / q_{n}\right)_{n \geq 0}$ denote the sequence of partial quotients of a real number $\xi$. Let $\eta$ be a positive integer. If $\xi$ is algebraic, then $q_{n+1} \leq q_{n}^{1+\eta}$ holds for any integer $n$ sufficiently large.

\section{Proofs of our MAin RESUlts}

Proof of Theorem 3.1. We follow the proof of Theorem 1 from 4, except that we use Theorem 4.1 instead of (13) and that we suitably apply Lemma [5.5 to get rid of the assumption (2). For completeness, we give the details of the argument.

Assume that $\xi$ is algebraic of degree $d$. For any positive integer $k$, set

$$
\xi_{k}:=[a_{0} ; a_{1}, \cdots, a_{n_{k}-1}, \underbrace{a_{n_{k}}, \ldots, a_{n_{k}+r_{k}-1}}_{\infty}] .
$$

Since the height of $\xi_{k}$ is at most $2 q_{n_{k}+r_{k}-1}^{2}$, the Liouville inequality (see e.g. [7, Corollary A.2) and Lemma 5.5 give us that

$$
\left|\xi-\xi_{k}\right| \gg q_{n_{k}+r_{k}-1}^{-2 d} \gg q_{n_{k}-1}^{-2 d} K_{r_{k}}\left(a_{n_{k}}, \ldots, a_{n_{k}+r_{k}-1}\right)^{-2 d} .
$$

Here and below, the constants implied by $\ll$ depend only on $\xi$. However, we infer from Lemmas 5.2, 5.3 and 5.5 that

$$
\begin{aligned}
\left|\xi-\xi_{k}\right| & \ll q_{n_{k}+\lambda_{k} r_{k}-1}^{-2} \\
& \ll q_{n_{k}-1}^{-2} K_{r_{k}}\left(a_{n_{k}}, \ldots, a_{n_{k}+r_{k}-1}\right)^{-2 d} K_{2 r_{k}}\left(a_{n_{k}}, \ldots, a_{n_{k}+2 r_{k}-1}\right)^{-\lambda_{k}+2 d} \\
& \ll q_{n_{k}-1}^{-2} K_{r_{k}}\left(a_{n_{k}}, \ldots, a_{n_{k}+r_{k}-1}\right)^{-2 d} 2^{-\lambda_{k} / 2}
\end{aligned}
$$

if $k$ is sufficiently large. A combination of the last two inequalities gives that

$$
\lambda_{k} \ll \log q_{n_{k}} .
$$


By the assumption (7), we then get that

$$
\limsup _{k \rightarrow \infty} \frac{\log \log q_{n_{k}}}{n_{k}^{\varepsilon+2 / 3}}=+\infty,
$$

a contradiction with Corollary 4.2 .

Proof of Theorem 3.2. For any $k \geq 0$, set

$$
K_{k}:=K_{r_{k}}\left(a_{n_{k}}, \ldots, a_{n_{k}+r_{k}-1}\right) .
$$

By assumption, there exist a positive real number $c$ and an infinite set of integers $\mathcal{K}_{1}$, ranged in increasing order, such that $\lambda_{k} \geq c n_{k}$ for any $k$ in $\mathcal{K}_{1}$.

The proof splits into two parts. Assume first that the sequence $\left(K_{k}\right)_{k \in \mathcal{K}_{1}}$ is bounded. Since the $K_{k}$ 's are non-negative integers, it follows that infinitely of them take the same value. Then, Lemma [5.3 implies that there exist a positive integer $r$, positive integers $b_{0}, \ldots, b_{r-1}$ and an infinite set $\mathcal{K}_{2}$ of positive integers such that

$$
r_{k}=r, \quad a_{n_{k}+j}=b_{j}, \quad(0 \leq j \leq r-1),
$$

for any $k$ in $\mathcal{K}_{2}$.

Let $\alpha$ denote the real number having the purely periodic continued fraction expansion with period $B=\left(b_{r-1}, \ldots, b_{0}\right)$, that is,

$$
\alpha=\left[b_{r-1} ; b_{r-2}, \ldots, b_{0}, b_{r-1}, \ldots, b_{0}, b_{r-1}, \ldots\right]=[B, B, \ldots, B, \ldots] .
$$

Then, $\alpha$ is a quadratic number. We need to introduce some more notation. Let us denote by $p_{n} / q_{n}$ (respectively, by $r_{n} / s_{n}$ ) the $n$-th convergent to $\xi$ (respectively, to $\alpha$ ). Then, for any $k$ in $\mathcal{K}_{2}$, set $P_{k}=p_{n_{k}+\lambda_{k} r_{k}-1}, Q_{k}=$ $q_{n_{k}+\lambda_{k} r_{k}-1}, P_{k}^{\prime}=p_{n_{k}+\lambda_{k} r_{k}-2}, Q_{k}^{\prime}=q_{n_{k}+\lambda_{k} r_{k}-2}$ and $S_{k}=s_{r \lambda_{k}-1}$.

By assumption, we already know that $\xi$ is irrational and not quadratic. Therefore, we assume that $\xi$ is algebraic and we aim at deriving a contradiction.

Let $k$ be in $\mathcal{K}_{2}$. By the theory of continued fractions, we have

$$
\left|Q_{k} \xi-P_{k}\right|<\frac{1}{Q_{k}} \text { and }\left|Q_{k}^{\prime} \xi-P_{k}^{\prime}\right|<\frac{1}{Q_{k}^{\prime}} .
$$

On the other hand, since by assumption

$$
\frac{P_{k}}{Q_{k}}=[a_{0} ; a_{1}, \cdots, a_{n_{k}-1}, \underbrace{B, B, \ldots, B}_{\lambda_{k}}],
$$

we get from the mirror formula (see Lemma [5.4) that

$$
\frac{Q_{k}}{Q_{k}^{\prime}}=[\underbrace{B, B, \ldots, B}_{\lambda_{k}}, a_{n_{k}-1}, \cdots, a_{1}] .
$$

Then, Lemma 5.2 implies

$$
\left|Q_{k}^{\prime} \alpha-Q_{k}\right|<\frac{Q_{k}^{\prime}}{S_{k}^{2}}
$$

and we a fortiori obtain that

$$
\lim _{\mathcal{K}_{2} \ni k \rightarrow \infty} \frac{Q_{k}}{Q_{k}^{\prime}}=\alpha
$$


Consider now the four linearly independent linear forms, whose coefficients are by assumption algebraic:

$$
\begin{aligned}
& L_{1}\left(X_{1}, X_{2}, X_{3}, X_{4}\right)=\xi X_{1}-X_{3}, \\
& L_{2}\left(X_{1}, X_{2}, X_{3}, X_{4}\right)=\xi X_{2}-X_{4}, \\
& L_{3}\left(X_{1}, X_{2}, X_{3}, X_{4}\right)=\alpha X_{2}-X_{1}, \\
& L_{4}\left(X_{1}, X_{2}, X_{3}, X_{4}\right)=X_{1} .
\end{aligned}
$$

Evaluating them on the quadruple $\left(Q_{k}, Q_{k}^{\prime}, P_{k}, P_{k}^{\prime}\right)$, it follows from (20) and (21) that

$$
\prod_{1 \leq j \leq 4}\left|L_{j}\left(Q_{k}, Q_{k}^{\prime}, P_{k}, P_{k}^{\prime}\right)\right|<\frac{1}{S_{k}^{2}}
$$

Let $M$ be an upper bound for the sequence $\left(q_{n}^{1 / n}\right)_{n \geq 1}$. We infer from Lemma 5.3 that $Q_{k} \leq(M+1)^{n_{k}+r \lambda_{k}}$ and $S_{k} \geq(\sqrt{2})^{r \lambda_{k}-2}$, for any positive integer $k$ in $\mathcal{K}_{2}$. It thus follows that

$$
S_{k} \geq(M+1)^{\left(\frac{\log \sqrt{2}}{\log (M+1)}\right)\left(r \lambda_{k}-2\right)} \geq Q_{k}^{\left(\frac{\log \sqrt{2}}{\log (M+1)}\right) \cdot\left(\frac{r \lambda_{k}-2}{n_{k}+r \lambda_{k}}\right)},
$$

for any positive integer $k$ in $\mathcal{K}_{2}$. In particular, we get from (23) and (8) that

$$
\prod_{1 \leq j \leq 4}\left|L_{j}\left(Q_{k}, Q_{k}^{\prime}, P_{k}, P_{k}^{\prime}\right)\right| \leq Q_{k}^{-\varepsilon}
$$

holds for some positive real number $\varepsilon$ and for $k$ large enough in $\mathcal{K}_{2}$.

It then follows from Theorem 5.1 that the points $\left(Q_{k}, Q_{k}^{\prime}, P_{k}, P_{k}^{\prime}\right)$ for $k$ in $\mathcal{K}_{2}$ lie in a finite number of proper subspaces of $\mathbb{Q}^{4}$. Thus, there exist a nonzero integer quadruple $\left(x_{1}, x_{2}, x_{3}, x_{4}\right)$ and an infinite set of distinct positive integers $\mathcal{K}_{3} \subset \mathcal{K}_{2}$ such that

$$
x_{1} Q_{k}+x_{2} Q_{k}^{\prime}+x_{3} P_{k}+x_{4} P_{k}^{\prime}=0,
$$

for any $k$ in $\mathcal{K}_{3}$. Dividing (24) by $Q_{k}^{\prime}$, we obtain

$$
x_{1} \frac{Q_{k}}{Q_{k}^{\prime}}+x_{2}+x_{3} \frac{P_{k}}{Q_{k}} \cdot \frac{Q_{k}}{Q_{k}^{\prime}}+x_{4} \frac{P_{k}^{\prime}}{Q_{k}^{\prime}}=0 .
$$

By letting $k$ tend to infinity along $\mathcal{K}_{3}$ in (25), we derive from (22) that

$$
x_{1} \alpha+x_{2}+\left(x_{3} \alpha+x_{4}\right) \xi=0 \text {. }
$$

Since $\xi$ is not quadratic, it a fortiori cannot lie in $\mathbb{Q}(\alpha)$. This implies that $x_{3} \alpha+x_{4}=0$ and, since $\alpha$ is irrational, it follows that $x_{3}=x_{4}=0$. Then, again by using that $\alpha$ is irrational, we get that $x_{1}=x_{2}=x_{3}=x_{4}=0$, which is a contradiction. This concludes the proof when the sequence $\left(K_{k}\right)_{k \in \mathcal{K}_{1}}$ is bounded.

Assume now that the sequence $\left(K_{k}\right)_{k \in \mathcal{K}_{1}}$ is unbounded. Then, there exists an infinite set $\mathcal{K}_{4}$ of integers, ranged in increasing order, such that the sequence $\left(K_{k}\right)_{k \in \mathcal{K}_{4}}$ increases to infinity.

Recall that $\left(p_{n} / q_{n}\right)_{n \geq 0}$ denotes the sequence of convergents to $\xi$ and that $M$ denotes an upper bound for the sequence $\left(q_{n}^{1 / n}\right)_{n \geq 0}$. Let $d$ be a positive integer. Let $k$ be in $\mathcal{K}_{4}$ and large enough in order that $\lambda_{k} \geq d+1$ and

$$
K_{k} \geq M^{2 d / c},
$$


with the constant $c$ defined at the beginning of the proof. Then, the real number $\xi$ is very close to the quadratic number

$$
\xi_{k}:=[a_{0} ; a_{1}, \cdots, a_{n_{k}-1}, \underbrace{a_{n_{k}}, \ldots, a_{n_{k}+r_{k}-1}}_{\infty}] .
$$

Define the polynomial

$$
\begin{aligned}
P_{k}(X):=\left(q_{n_{k}-2} q_{n_{k}+r_{k}-1}-q_{n_{k}-1} q_{n_{k}+r_{k}-2}\right) X^{2} & \\
-\left(q_{n_{k}-2} p_{n_{k}+r_{k}-1}-q_{n_{k}-1} p_{n_{k}+r_{k}-2}\right. & \left.+p_{n_{k}-2} q_{n_{k}+r_{k}-1}-p_{n_{k}-1} q_{n_{k}+r_{k}-2}\right) X \\
& +\left(p_{n_{k}-2} p_{n_{k}+r_{k}-1}-p_{n_{k}-1} p_{n_{k}+r_{k}-2}\right),
\end{aligned}
$$

and observe that $P_{k}\left(\xi_{k}\right)=0$. For any positive integer $k$, we infer from Rolle's Theorem and Lemma 5.2 that

$$
\begin{aligned}
\left|P_{k}(\xi)\right| & =\left|P_{k}(\xi)-P_{k}\left(\xi_{k}\right)\right| \ll q_{n_{k}-1} q_{n_{k}+r_{k}-1}\left|\xi-\xi_{k}\right| \\
& \ll q_{n_{k}-1} q_{n_{k}+r_{k}-1} q_{n_{k}+\lambda_{k} r_{k}-1}^{-2},
\end{aligned}
$$

since the first $n_{k}+\lambda_{k} r_{k}-1$ partial quotients of $\xi$ and $\xi_{k}$ are the same. Here and below, the constants implied in $\ll$ depend at most on $\xi$ and on $d$, but they are independent on $k$. Now, it follows from Lemma [5.5] that

$$
q_{n_{k}+\lambda_{k} r_{k}-1} \geq q_{n_{k}-1} K_{k}^{\lambda_{k}}
$$

thus, by (26) and by Lemma 5.5 again, we get

$$
\left|P_{k}(\xi)\right| \ll K_{k}^{1-2 \lambda_{k}} \ll K_{k}^{-\lambda_{k}-d} \ll\left(M^{2 n_{k}} K_{k}\right)^{-d} \ll\left(q_{n_{k}-1} q_{n_{k}+r_{k}-1}\right)^{-d},
$$

since $\lambda_{k} \geq d+1$. Recalling that $\xi$ is irrational and not quadratic, it then follows from the Liouville inequality (see e.g. [7, Theorem A.1) that $\xi$ cannot be algebraic of degree smaller than $d$. Since $d$ is arbitrary, this concludes the proof when the sequence $\left(K_{k}\right)_{k \in \mathcal{K}_{1}}$ is bounded.

Proof of Corollary 3.3. Let us consider the quasi-periodic continued fraction

$$
\xi=[a_{0} ; a_{1}, \ldots, a_{n_{0}-1}, \underbrace{a_{n_{0}}, \ldots, a_{n_{0}+r_{0}-1}}_{\lambda_{0}}, \underbrace{a_{n_{1}}, \ldots, a_{n_{1}+r_{1}-1}}_{\lambda_{1}}, \ldots],
$$

satisfying the assumption of the corollary, and suppose that we have

$$
\liminf _{k \rightarrow \infty} \frac{\lambda_{k+1}}{\lambda_{k}}>1
$$

For $k \geq 1$, we get that

$$
n_{k}=n_{0}+\sum_{j=0}^{k-1} r_{j} \lambda_{j} .
$$

Moreover, we infer from (27) that there exist positive real numbers $\delta$ and $M$ such that

$$
\lambda_{j}<\frac{M \lambda_{k}}{(1+\delta)^{k-j}}
$$


for any $j<k$ with $k$ large enough. Since the sequence $\left(r_{k}\right)_{k \geq 0}$ is bounded, there exists a positive real number $r$ such that

$$
n_{k}<n_{0}+r \lambda_{k} \sum_{j \geq 1} \frac{M}{(1+\delta)^{j}}
$$

and thus

$$
\limsup _{k \rightarrow \infty} \frac{\lambda_{k}}{n_{k}} \geq \frac{1}{n_{0}+r M\left(\sum_{j \geq 1}(1+\delta)^{-j}\right)}>0,
$$

for $k$ large enough. Applying Theorem 3.2, this concludes the proof.

Proof of Theorem 3.4. For any $k \geq 0$, set

$$
K_{k}:=K_{r_{k}}\left(a_{n_{k}}, \ldots, a_{n_{k}+r_{k}-1}\right) .
$$

In view of Corollary 3.3 there is no restriction in assuming that the sequence $\left(K_{k}\right)_{k \geq 0}$ is unbounded. Then, there exists an infinite set $\mathcal{K}_{5}$ of integers, ranged in increasing order, such that the sequence $\left(K_{k}\right)_{k \in \mathcal{K}_{5}}$ increases to infinity and such that, for any $k$ in $\mathcal{K}_{5}$ and any integer $j$ with $0 \leq j<k$, we have $K_{j}<K_{k}$.

Let $k$ be in $\mathcal{K}_{5}$. The real number $\xi$ is very close to the quadratic number

$$
\xi_{k}:=[a_{0} ; a_{1}, \cdots, a_{n_{k}-1}, \underbrace{a_{n_{k}}, \ldots, a_{n_{k}+r_{k}-1}}_{\infty}] .
$$

Let $j_{k}$ be the largest integer $<n_{k}$ such that $a_{j_{k}} \neq a_{j_{k}+r_{k}}$. Choosing $k$ sufficiently large, $j_{k}$ is well defined, since $\left(a_{k}\right)_{k \geq 0}$ is not ultimately periodic. Observe that

$$
\xi_{k}:=[a_{0} ; a_{1}, \cdots, a_{j_{k}}, \underbrace{a_{j_{k}+1}, \ldots, a_{j_{k}+r_{k}}}_{\infty}] .
$$

Let $\eta \leq 1$ be a positive real number. We then assume that $\xi$ is algebraic and we proceed as in the proof of Theorem 2 from [1].

Define the polynomial

$$
\begin{aligned}
P_{k}(X):= & \left(q_{j_{k}-1} q_{j_{k}+r_{k}}-q_{j_{k}} q_{j_{k}+r_{k}-1}\right) X^{2} \\
& -\left(q_{j_{k}-1} p_{j_{k}+r_{k}}-q_{j_{k}} p_{j_{k}+r_{k}-1}+p_{j_{k}-1} q_{j_{k}+r_{k}}-p_{j_{k}} q_{j_{k}+r_{k}-1}\right) X \\
& +\left(p_{j_{k}-1} p_{j_{k}+r_{k}}-p_{j_{k}} p_{j_{k}+r_{k}-1}\right),
\end{aligned}
$$

and observe that $P_{k}\left(\xi_{k}\right)=0$. For any positive integer $k$ in $\mathcal{K}_{5}$, we infer from Rolle's Theorem and Lemma 5.2 that

$$
\left|P_{k}(\xi)\right|=\left|P_{k}(\xi)-P_{k}\left(\xi_{k}\right)\right| \ll q_{j_{k}} q_{j_{k}+r_{k}}\left|\xi-\xi_{k}\right| \ll q_{j_{k}} q_{j_{k}+r_{k}} q_{j_{k}+\lambda_{k} r_{k}}^{-2},
$$

since the first $j_{k}+\lambda_{k} r_{k}$ partial quotients of $\xi$ and $\xi_{k}$ are the same.

Since $\xi$ is assumed to be algebraic, it follows from Lemma 5.6 that

$$
\left|\left(q_{j_{k}-1} q_{j_{k}+r_{k}}-q_{j_{k}} q_{j_{k}+r_{k}-1}\right) \xi-\left(q_{j_{k}-1} p_{j_{k}+r_{k}}-q_{j_{k}} p_{j_{k}+r_{k}-1}\right)\right| \ll q_{j_{k}} q_{j_{k}+r_{k}}^{-1+\eta}
$$

and

$\left|\left(q_{j_{k}-1} q_{j_{k}+r_{k}}-q_{j_{k}} q_{j_{k}+r_{k}-1}\right) \xi-\left(p_{j_{k}-1} q_{j_{k}+r_{k}}-p_{j_{k}} q_{j_{k}+r_{k}-1}\right)\right| \ll q_{j_{k}}^{-1+\eta} q_{j_{k}+r_{k}}$, 
if $k$ in $\mathcal{K}_{5}$ is large enough. Furthermore, we have as well the obvious upper bound

$$
\left|q_{j_{k}-1} q_{j_{k}+r_{k}}-q_{j_{k}} q_{j_{k}+r_{k}-1}\right| \leq q_{j_{k}} q_{j_{k}+r_{k}}
$$

Consider now the four linearly independent linear forms with algebraic coefficients:

$$
\begin{aligned}
& L_{1}\left(X_{1}, X_{2}, X_{3}, X_{4}\right)=\xi^{2} X_{1}-\xi\left(X_{2}+X_{3}\right)+X_{4}, \\
& L_{2}\left(X_{1}, X_{2}, X_{3}, X_{4}\right)=\xi X_{1}-X_{2}, \\
& L_{3}\left(X_{1}, X_{2}, X_{3}, X_{4}\right)=\xi X_{1}-X_{3}, \\
& L_{4}\left(X_{1}, X_{2}, X_{3}, X_{4}\right)=X_{1} .
\end{aligned}
$$

Evaluating them on the quadruple

$$
\begin{gathered}
\underline{z}_{k}:=\left(q_{j_{k}-1} q_{j_{k}+r_{k}}-q_{j_{k}} q_{j_{k}+r_{k}-1}, q_{j_{k}-1} p_{j_{k}+r_{k}}-q_{j_{k}} p_{j_{k}+r_{k}-1},\right. \\
\left.p_{j_{k}-1} q_{j_{k}+r_{k}}-p_{j_{k}} q_{j_{k}+r_{k}-1}, p_{j_{k}-1} p_{j_{k}+r_{k}}-p_{j_{k}} p_{j_{k}+r_{k}-1}\right),
\end{gathered}
$$

we find that

$$
\Pi:=\prod_{1 \leq j \leq 4}\left|L_{j}\left(\underline{z}_{k}\right)\right| \ll\left(q_{j_{k}} q_{j_{k}+r_{k}}\right)^{2+\eta} q_{j_{k}+\lambda_{k} r_{k}}^{-2} .
$$

Now, it follows from the last assertion of Lemma [5.5 that

$$
q_{j_{k}+\lambda_{k} r_{k}} \geq q_{j_{k}}\left(K_{k} / 2\right)^{\lambda_{k}}
$$

thus, by Lemma 5.5 again,

$$
\Pi \ll q_{j_{k}}^{2+2 \eta}\left(2 K_{k}\right)^{2+\eta}\left(K_{k} / 2\right)^{-2 \lambda_{k}} \ll K_{k}^{2(1+\eta)\left(\lambda_{1}+\ldots+\lambda_{k-1}+k\right)}\left(K_{k} / 2\right)^{-2 \lambda_{k}} .
$$

Using hypotheses (10) and choosing $\eta$ small enough, we infer from the preceding inequality that there exists a positive real number $\varepsilon$ such that

$$
\Pi \ll K_{k}^{-2\left(\lambda_{1}+\ldots+\lambda_{k-1}+k\right) \varepsilon} \ll\left(q_{j_{k}} q_{j_{k}+r_{k}}\right)^{-\varepsilon} .
$$

It then follows from Theorem 5.1 that the points $\underline{z}_{k}$ for $k$ in $\mathcal{K}_{5}$ lie in a finite number of proper subspaces of $\mathbb{Q}^{4}$. Thus, there exist a nonzero integer quadruple $\left(x_{1}, x_{2}, x_{3}, x_{4}\right)$ and an infinite set of distinct positive integers $\mathcal{K}_{6} \subset$ $\mathcal{K}_{5}$ such that

$$
\begin{gathered}
x_{1}\left(q_{j_{k}-1} q_{j_{k}+r_{k}}-q_{j_{k}} q_{j_{k}+r_{k}-1}\right)+x_{2}\left(q_{j_{k}-1} p_{j_{k}+r_{k}}-q_{j_{k}} p_{j_{k}+r_{k}-1}\right) \\
+x_{3}\left(p_{j_{k}-1} q_{j_{k}+r_{k}}-p_{j_{k}} q_{j_{k}+r_{k}-1}\right)+x_{4}\left(p_{j_{k}-1} p_{j_{k}+r_{k}}-p_{j_{k}} p_{j_{k}+r_{k}-1}\right)=0 .
\end{gathered}
$$

for any $k$ in $\mathcal{K}_{6}$. We then argue exactly as in the proof of Theorem 2 from 1. This is made possible by our choice of $j_{k}$. We then reach a contradiction, which concludes the proof of our theorem.

\section{REFERENCES}

[1] B. Adamczewski, Y. Bugeaud, On the complexity of algebraic numbers II. Continued fractions, Acta Math., to appear.

[2] B. Adamczewski, Y. Bugeaud, Palindromic continued fractions, Preprint, 2005.

[3] J.-P. Allouche, Nouveaux résultats de transcendance de réels à développement non aléatoire, Gaz. Math. 84 (2000) 19-34.

[4] A. Baker, Continued fractions of transcendental numbers, Mathematika 9 (1962) 1-8.

[5] A. Baker, On Mahler's classification of transcendental numbers, Acta Math. 111 (1964) $97-120$.

[6] E. Bombieri, A. J. van der Poorten, Some quantitative results related to Roth's theorem, J. Austral. Math. Soc. Ser. A 45 (1988), 233-248.

[7] Y. Bugeaud, Approximation by algebraic numbers, Cambridge Tracts in Mathematics 160, Cambridge, 2004. 
[8] J. L. Davison, Quasi-Periodic Continued Fractions, Preprint, 2005.

[9] H. Davenport, K. F. Roth, Rational approximations to algebraic numbers, Mathematika 2 (1955), 160-167.

[10] J.-H. Evertse, The number of algebraic numbers of given degree approximating a given algebraic number. In: Analytic number theory (Kyoto, 1996), 53-83, London Math. Soc. Lecture Note Ser. 247, Cambridge Univ. Press, Cambridge, 1997.

[11] A. Ya. Khintchine, Continued Fractions (in Russian), Gosudarstv. Izdat. Tehn.-Teor. Lit., Moscow-Leningrad, 2nd edition, 1949.

[12] S. Lang, Introduction to Diophantine Approximations, Addison-Wesley Publishing Co., Reading, Mass.-London-Don Mills, Ont., 1966.

[13] W. J. LeVeque, Topics in number theory. Vol. 1, 2, Addison-Wesley Publishing Co., Inc., Reading, Mass., 1956.

[14] J. Liouville, Sur des classes très étendues de quantités dont la valeur n'est ni algébrique, ni même réductible à des irrationelles algébriques, C. R. Acad. Sci. Paris 18 (1844) 883-885; 910-911.

[15] E. Maillet, Introduction à la théorie des nombres transcendants et des propriétés arithmétiques des fonctions, Gauthier-Villars, Paris, 1906.

[16] M. Mignotte, Quelques remarques sur l'approximation rationnelle des nombres algébriques, J. reine angew. Math. 268/269 (1974), 341-347.

[17] O. Perron, Die Lehre von den Kettenbrüchen, Teubner, Leibzig, 1929.

[18] K. F. Roth, Rational approximations to algebraic numbers, Mathematika 2 (1955) 1-20; corrigendum 168.

[19] W. M. Schmidt, Norm form equations, Ann. of Math. (2), 96 (1972) 526-551.

[20] W. M. Schmidt, Diophantine approximation, Lecture Notes in Mathematics $\mathbf{7 8 5}$, Springer, Berlin, 1980.

[21] J. Shallit, Real numbers with bounded partial quotients: a survey, Enseign. Math. 38 (1992) 151-187.

[22] M. Waldschmidt, Un demi-siècle de transcendance. In: Development of mathematics 1950-2000, pp. 1121-1186, Birkhäuser, Basel, 2000.

Boris Adamczewski

CNRS, Institut Camille Jordan

Université Claude Bernard Lyon 1

Bât. Braconnier, 21 avenue Claude Bernard

69622 VILLEURBANNE Cedex

FRANCE

Boris.Adamczewski@math .univ-lyon1.f
Yann Bugeaud Université Louis Pasteur U. F. R. de mathématiques 7 , rue René Descartes 67084 STRASBOURG Cedex FRANCE 